\title{
Acknowledgement of Reviewers
}

We gratefully acknowledge the work of reviewers for Journal of Plant Diseases and Protection. The standards of scientific publishing are upheld by peer review and the work of our reviewers. We thank the many scientists who have given of their time and expertise to offer their judgements and to help authors in improving manuscripts during the year 2013.

Cornel Adler, Berlin, Germany

Ana Alfaro-Fernandez, Valencia, Spain

Randal Anderson, Brookings, SD, USA

Christos G Athanassiou, Volos, Greece

Dirk Uwe Bellstedt, Stellenbosch, South Africa

Natasza Borodynko, Poznan, Poland

Laura Breitsameter, Göttingen, Germany

François Brun, Toulouse, France

Christian Bruns, Witzenhausen, Germany

Balu Chopade, Pune, India

Fabrizio Cillo, Bari, Italy

Robert Czaikowski, Gdansk, Poland

Amer Dababat, Ankara, Turkey

Matthias Daub, Elsdorf, Germany

Vincenzo De Feo, Salerno, Italy

Yves Dessaux, Gif-sur-Yvette, France

Revel Drummond, Auckland, New Zealand

Robert Farrar, Beltsville, USA

Denis Faure, Gif-sur-Yvette, France

Maria Finckh, Witzenhausen, Germany

Henryk Flachowsky, Dresden, Germany

Karl-Heinz Gartemann, Bielefeld, Germany

Arnaud Gauffreteau, Grignon, France

Seyed MT Ghaffary, Dezfoul, Iran

Jean-Claude Grégoire, Brussels, Belgium

Rita Grosch, Großbeeren, Germany

Raul NC Guedes, Viçosa, Brazil

Dennis A Halterman, Madison, WI, USA

Katerina Hamouzová, Prague, Czech Republic

Inge M Hanssen, St.-Katelijne-Waver, Belgium

Lili Huang, Yangling, China

Marcello Iriti, Milan, Italy

Toru Iwanami, Tsukuba, Japan

Heikki Jalli, Jokioinen, Finland

Anthony P James, Brisbane, Australia

Kai-Uwe Katroschan, Gülzow, Germany

George Kennedy, Raleigh, NC, USA

Wolfgang H Kirchner, Bochum, Germany

Beata Komorowska, Skierniewice, Poland

Hermann-Josef Krauthausen, Neustadt, Germany

Kristian Kristensen, Tjele, Denmark

Thomas Kühne, Quedlinburg, Germany

Anders Kvarnheden, Uppsala, Sweden

Leonor Leandro, Ames, IA, USA

Miguel A Martínez-Téllez, Hermosillo, Mexico

Marta Martini, Udine, Italy
Dario Massa, Limburger Hof, Germany

Michael Meissle, Zürich, Switzerland

Daniel Molitor, Belvaux, Luxembourg

Enrique Moriones, Málaga, Spain

Gloria Mosquera, Cali, Colombia

Gary Munkvold, Ames, Iowa, USA

Kristine T Nemec, Brookings, SD, USA

Henry Ngugi, México, México

John Obrycki, Lexington, KY, USA

Erich-Christian Oerke, Bonn, Germany

Ely Oliveira Garcia, Halle, Germany

Euro Pannacci, Perugia, Italy

Daisy Perez-Brito, Mérida, México

Gerhard Pietersen, Pretoria, South Africa

Frank Rabenstein, Quedlinburg, Germany

Katja Richert, Braunschweig, Germany

Ellen Richter, Braunschweig, Germany

Jordi Riudavets, Cabrils, Spain

Vittorio Rossi, Piacenza, Italia

Craig S Rothrock, Fayetteville, AR, USA

Joachim Schiemann, Quedlinburg, Germany

Bernd Schneider, Dossenheim, Germany

Jörg Schubert, Quedlinburg, Germany

Jürgen Schwarz, Kleinmachnow, Germany

Erich Seemüller, Dossenheim, Germany

Martin Shapiro, Beltsville, USA

Mette Sønderskov, Slagelse, Denmark

Rajagopalbabu Srinivasan, Tifton, GA, USA

Gerd Stammler, Limburger Hof, Germany

Paul WJ Taylor, Melbourne, Australia

Thomas Thieme, Groß Lüsewitz, Germany

Tim C Thoden, Freiburg, Germany

Lena Ulber, Braunschweig, Germany

Jens-Georg Unger, Braunschweig, Germany

Jacquie van der Waals, Pretoria, South Africa

Irene Vloutoglou, Athens, Greece

Susanne Vogelgsang, Zürich, Switzerland

Zbigniew Weber, Poznan, Poland

Jon West, Rothamsted, UK

Andreas Westphal, Braunschweig, Germany

Mark Winter, Göttingen, Germany

Xiulan Xu, Columbus, OH, USA

Takashi Yamada, Hiroshima, Japan

Li Yang, Chapel Hill, NC, USA

Mingpei You, Perth, Australia 